\title{
The role of leucocytes in the acetyl salicylic acid (aspirin) induced nitric oxide synthesis in the production of interferon- $\alpha$, a potent inhibitor of platelet aggregation and a thrombolytic agent
}

\author{
Mau Bhattacharyya - Soumendra K. Karmohapatra • \\ Gorachand Bhattacharya - Rabindra Bhattacharya • \\ A. Kumar Sinha
}

Published online: 13 March 2009

(C) Springer Science+Business Media, LLC 2009

\section{Erratum to: J Thromb Thrombolysis DOI 10.1007/s11239-008-0283-1}

The authors wish to make the following corrections.

Figure 2: The letters "A" to "I" (A, B, C, D, E, F, G, I) were omitted in the horizontal axis of the Figure 2.

Figure 9: In the title of Figure 9 it should be "IFN- $\alpha$ " instead of "aspirin." The title should read "Effect of injection of IFN- $\alpha$ in the circulation on the lysis of thrombi in the coronary artery in mice."

The online version of the original article can be found under doi:10.1007/s11239-008-0283-1.

M. Bhattacharyya · S. K. Karmohapatra - A. K. Sinha ( $\square)$

Sinha Institute of Medical Science \& Technology,

288, Kendua Main Road, Garia, Calcutta 700084, India

e-mail: asruksinha@yahoo.com

G. Bhattacharya

National Medical College, Calcutta 700014, India

R. Bhattacharya

Calcutta Medical College \& Hospital, Calcutta 700072, India

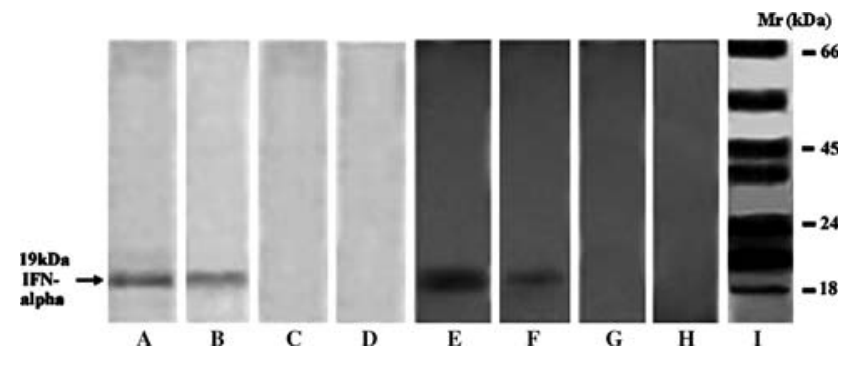

Fig. 2 Immunoblot of the dialyzed and concentrated supernatant from aspirin treated leucocyte suspension. Supernatant from the leucocyte suspension treated with $80 \mu \mathrm{M}$ was prepared and concentrated. The presence of IFN- $\alpha$ in the supernatant was carried out as described in the "Materials and methods" section. The results shown here is a typical representative of at least 10 other experiments using blood from 10 different donors. Lane A = SDS-polyacrylamide gel electrophoresis of pure IFN- $\alpha(11.56 \mathrm{pmol})$ stained with coomassie brilliant blue. Lane B = SDS-polyacrylamide gel electrophoresis of incubated supernatant (leukocytes + Aspirin) with coomassie brilliant blue. Lane C = SDS-polyacrylamide gel electrophoresis of only leucocyte supernatant stained with coomassie brilliant blue. Lane $\mathrm{D}=$ SDS-polyacrylamide gel electrophoresis of incubated supernatant (leukocytes + Aspirin + NAME) with coomassie brilliant blue. Lane $\mathrm{E}=$ Immunoblotted authentic IFN $\alpha$ after nitrocellulose membrane transfer. Lane $\mathrm{F}=$ Immunoblotted incubated supernatant (leucocytes + Aspirin) after nitrocellulose membrane transfer (as shown in lane B). Lane $\mathrm{G}=$ Immunoblotted leukocyte supernatant after nitrocellulose membrane transfer (as shown in lane C). Lane $\mathrm{H}=$ Immunoblotted incubated supernatant (leucocytes + Aspirin + NAME) after nitrocellulose membrane transfer (as shown in lane D). Lane I = Molecular weight markers stained with coomassie brilliant blue 


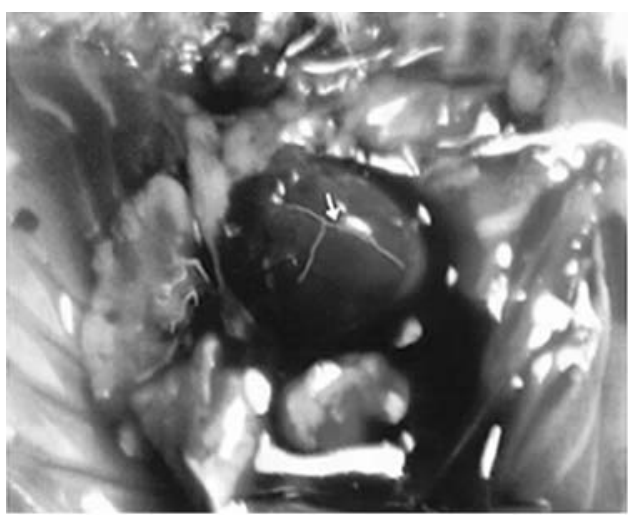

A

Fig. 9 Effect of injection of IFN- $\alpha$ in the circulation on the lysis of thrombi in the coronary artery in mice. a Coronary thrombosis was developed by injecting ADP through the tail vein of mice. White arrow indicates the formation of platelet thrombi in the coronary artery as white streak, as seen in postmortem examination of the heart

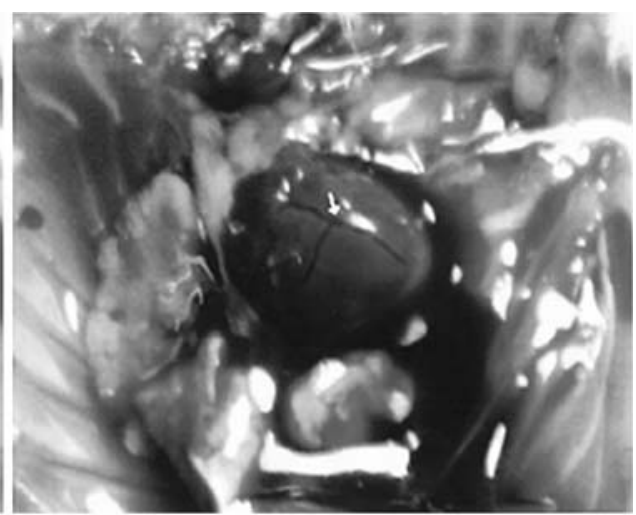

B

by light microscopy. b The animal received injection of $800 \mathrm{pmol} / \mathrm{kg}$ body weight IFN- $\alpha$ in the circulation within 5 min after the injection of ADP $(1.5 \mu \mathrm{mol} / \mathrm{g}$ body weight $)$ in the tail vein of the animal. The postmortem of the heart showed the clearing of the platelet thrombi (white arrow) as seen by light microscopy 